VIII ${ }^{\text {èmes }}$ Journées Nationales Génie Civil - Génie Côtier, Compiègne, 7-9 septembre 2004

\title{
Effet de la butée de pied posée à faible profondeur sur la stabilité de talus de protection
}

\author{
Hafid Tabet ${ }^{(\mathrm{a})}$, Jérôme Brossard ${ }^{(\mathrm{b})}$, \\ Michel Colinet ${ }^{(\mathrm{c})}$, Bruno Jacinthe ${ }^{(\mathrm{d})}$ \\ ${ }^{(a)}$ PAST, université du Havre, hafid.tabet@univ-lehavre.fr \\ ${ }^{(b)} H D R$, université du Havre, jerome.brossard@univ-lehavre.fr \\ ${ }^{(c)}$ Chef de projet, CREOCEAN, michel.colinet@creocean.fr \\ ${ }^{(d)}$ Ingénieur structure, CETEM Antilles,
}

\section{Résumé}

Un programme d'étude a fait l'objet d'une série d'essais en modèle $2 \mathrm{D}$ en vue de rechercher les meilleures configurations possibles de talus à faibles profondeurs soumis à des houles régulières déferlantes. Plus de seize types de conception de butée ont été testées en faisant varier la composition de cette dernière ainsi que le niveau de son soubassement. L'analyse des résultats confirme l'effet de la pente ainsi que l'érosion de la butée de pied sur la stabilité du talus. Les points suivants ont été identifiés. Le seuil d'instabilité entraînant la ruine de l'ouvrage baisse avec le niveau d'eau en pied de talus. Ni l'augmentation relative de la taille des blocs de carapace, ni le "'gabionnage"' des blocs de butée ne donnent lieu à une amélioration sensible du seuil d'instabilité. Il est conclu ainsi que dans de tels cas l'augmentation substantielle de la taille des blocs en butée n'est pas toujours la solution pour assurer la stabilité de cette dernière. L'érosion du pied est souvent due à la présence éventuelle de discontinuités verticales dans la pente du talus.

\begin{abstract}
$\underline{\text { Abstract }}$
The present study summarizes the results of a series of embankment 2D model tests in order to analyse the stability of the toe. More than sixteen types of the structure were tested on shallow water with regular breaking waves. The study confirms the slope effect and the sensitivity of the toe scouring on the embankment stability. Neither the over sizing nor the linkage of the toe blocks improve the stability of the whole embankment. It was concluded that the size of the blocks does not provide a guarantee of the stability because of the scouring due to the possible presence of vertical discontinuity on the structure slope.
\end{abstract}

Mots-clés: Brise-lames, digues, butée de pied, érosion, déferlement, stabilité

\section{Introduction}

Un programme d'étude réalisée au sein du Laboratoire de Mécanique, Physique et Géosciences de l'université du Havre en partenariat avec le Conseil Général de la Martinique et le bureau d'études CREOCEAN, a fait l'objet de séries d'essais en canal à houle en vue de rechercher les meilleures configurations possibles de talus pour la protection côtière sur des sites à pentes relativement raides (pente de 7\%) soumis à des houles déferlantes de grandes périodes ( $\mathrm{T}=14 \mathrm{~s}$ à $16 \mathrm{~s})$.

L'étude a porté sur des configurations de talus avec différentes pentes (2/1, 3/2 et 4/3) et deux niveaux d'eau $\left(5,00 \mathrm{~m}\right.$ et $3,75 \mathrm{~m}$ en nature). Deux échelles $\left(1 / 53^{\text {ème }}\right.$ et $\left.1 / 60^{\text {ème }}\right)$ ont servi à tester la stabilité du talus protégé par des blocs en béton de forme proche du parallélépipède. 
La position de la butée, les discontinuités de la pente dues à la présence de cette dernière sont des paramètres importants pour la stabilité de tels ouvrages à faibles profondeurs soumis à des houles déferlantes.

Différentes configurations de butées ont été testées avec plusieurs types de conception du soubassement. La sollicitation de chacune de ces configurations avec des houles croissantes en périodes et en amplitudes a montré la grande sensibilité de l'instabilité de la butée sur la ruine de l'ouvrage.

L’objectif du présent papier est de contribuer à la compréhension de ces phénomènes d'érosion de pied de talus et l'impact sur la stabilité de l'ensemble de l'ouvrage. Un soin particulier à la conception de la butée et la recherche d'une protection renforcée contre l'érosion en pied doivent à chaque fois être recherchés.

\section{Etat des connaissances}

La stabilité de la butée de pied de la digue à talus est importante. Sa conception requiert une attention particulière. Elle peut être sujet à des déformations tant par les forces hydrodynamiques qui la sollicitent que par les mouvements des fonds meubles sur lesquels elle s'appuie.

USACE (2001) recommande ainsi pour assurer la stabilité du talus, d'étendre les blocs de carapace jusqu'à un niveau de l'ordre de 1,5 fois la houle de projet au dessous du niveau des plus basses mers lorsque la structure est posée sur des fonds supérieurs à 1,5H. Dans le cas contraire, les blocs de carapace doivent être posés jusqu'au fond; mais les conditions de butée de pied à l'interface entre le talus de l'ouvrage et la pente des fonds demeure une zone de stabilité critique. Cette dernière doit donc être conçue avec beaucoup de soins.

Différents auteurs se sont intéressés à ce problème. Citons entres autres, Sawaragi (1966) et Arneborg (1995) qui se sont intéressés tout particulièrement au problème d'érosion du pied de talus. Leurs essais respectivement sur modèle physique et sur modèle numérique ont révélé l'influence de la réflexion sur les conditions hydrodynamiques sollicitant l'ouvrage.

Sumer et Fredsoe (2000) ont étudié en canal à houle deux sections de digue à talus avec deux pentes différentes soumises à des houles régulières puis à des houles irrégulières. Ils ont pu montré ainsi qu'alternativement des zones d'érosion et de sédimentation apparaissaient devant le talus sur des lignes parallèles à la digue, avec des profondeurs d'érosion plus réduites lorsque la digue est sollicitée par des houles aléatoires.

Le risque potentiel de l'érosion devant les ouvrages en mer et une synthèse sur l'état des connaissances sur le sujet ont fait en particulier l'objet d'un important programme de recherche européen s'étalant sur une période de trois ans (1997-2000), appelé ''Scour around coastal structure, SCARCOST',

Les résultats des travaux ont été résumés par Summer B. M. et al (2001), précisant que : - l'évolution de l'érosion devant les ouvrages types digue à talus est, comme pour les structures verticales, fonction de la cambrure de la houle et de la pente du talus

- Pour une même cambrure de la houle l'érosion diminue avec la pente du talus. De même, la profondeur d'érosion est 20 à 30\% plus importante si le sédiment est mis en suspension (sédiment fin) au lieu d’être charrié (sédiment grossier)

Néanmoins et même si le mécanisme de dégradation général des digues à talus est maintenant relativement bien compris, il n'y a apparemment pas d'étude disponible dans la littérature existante traitant le problème de l'érosion de la butée de pied et son effet sur la stabilité du talus d'une manière systématique.

Nous tentons dans le présent papier d'apporter notre contribution à l'analyse de la tenue de la butée de pied et ses effets sur la stabilité d’ensemble à travers les essais 2D réalisés dans 
le cas d'un talus de protection posé sur une souille rigide ou composée de matériaux meubles, soumis en particulier à des houles longues déferlant sur l'ouvrage.

\section{Les moyens d'essai}

Le modèle physique est installé dans un canal à houle de $10 \mathrm{~m}$ de long, 0,49 $\mathrm{m}$ de large et 0,48 $\mathrm{m}$ de profondeur. La partie centrale de $6 \mathrm{~m}$ de long est vitrée (parois latérales et fond) pour permettre les visualisations. Les extrémités du canal, de $2 \mathrm{~m}$ de long chacune, sont opaques. Pour permettre l'enregistrement vidéo du comportement des blocs nous avons dû installer le talus dans une partie vitrée du canal comme indiqué sur la figure 1 . La distance entre le batteur et le pied de talus était donc de 6,4 m.

Les amplitudes de houle prévues (jusqu'à 6,5m nature) nous ont conduit à choisir une hauteur d'eau maximale de $0,35 \mathrm{~m}$ dans le canal. Deux échelles de modèle $2 \mathrm{D}, 1 / 53^{\text {ème }}$ et $1 / 60^{\text {ème }}$, ont été utilisées lors des essais objet de la présente étude.

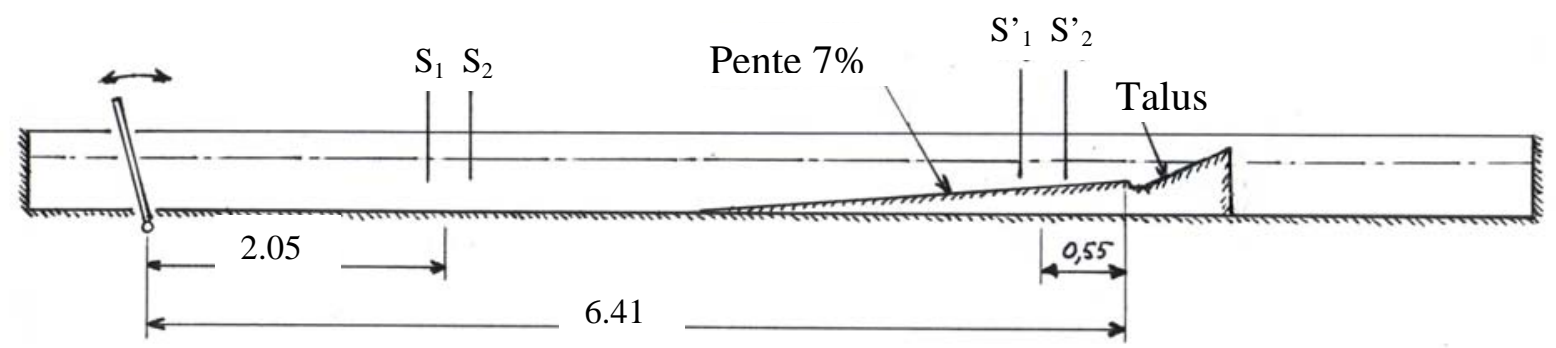

Figure 1 - Equipement du canal

Compte tenu des équipements disponibles, la houle générée dans le canal est monochromatique.

Même si les essais de stabilité des ouvrages en mer se font de plus en plus en houle aléatoire, la littérature existante fait apparaître certaines équivalences entre les sollicitations au moyen des houles régulières ou des houle aléatoires. Des travaux réalisés par certains chercheurs donnent des correspondances entre la hauteur $\mathrm{H}$ d'un train d'ondes monochromatiques sollicitant des ouvrages de type digue à talus et celle donnée dans le cas d'une houle aléatoire. Les dommages associés à des houles régulières seraient équivalents à ceux donnés par des houles irrégulières en prenant pour hauteur équivalente la valeur de $\mathrm{H}_{1 / 5}$ (Tanimoto et al, 1982), $\mathrm{H}_{1 / 10}$ (SPM, 1984), $\mathrm{H}_{1 / 20}$ (Jensen et al, 1996). Les différences dans les évaluations proviennent probablement selon Jensen des techniques utilisées dans chacun de ces cas.

Dans le cas présent et pour chaque test, la houle a été mesurée à deux endroits: au large et à proximité de l'ouvrage (figure1). La zone au large correspond à la partie du canal à profondeur constante. Les mesures de dénivellation sont réalisées par des sondes résistives. Régulièrement, au moins deux fois par jour (s'il n'y a pas de changement de hauteur d'eau), l'ensemble de la chaîne de mesure (sondes, conditionneurs et carte d'acquisition) est étalonné.

Par ailleurs, la digue provoque une réflexion non négligeable. Pour connaître la hauteur des vagues arrivant sur l'ouvrage la séparation de l'onde incidente et de l'onde réfléchie est faite au moyen de la méthode de Goda et Suzuki (1976).

La discrimination des ondes incidente et réfléchie permet d'estimer le coefficient de réflexion. Nous avons développé, sous $L A B V I E W$, des logiciels d'acquisition et de traitement 
adaptés à la méthode. Les quatre signaux d'évolution du niveau de la surface libre permettant de calculer les hauteurs de vague incidente et réfléchie sont enregistrés pour chaque test.

A noter enfin que les essais 2D se limitent à la simulation des effets des mouvements dans le profil du talus dus à l'incidence normale de la houle. Or une part non négligeable de l'érosion du pied de talus provient des courants latéraux Fredsoe et al (1997) ont étudié en laboratoire cette érosion autour du musoir de la digue à talus

\section{Préparation du modèle}

La réflexion provoque des ventres d'oscillation de la surface libre. Le premier, du côté de l'ouvrage, est situé à environ une demi longueur d'onde de celui ci dans la zone où la profondeur diminue (pente de $7 \%$ ). Cette diminution provoque, par effet de shoaling, une augmentation de la hauteur des vagues. Ces deux effets déterminent le déclenchement du déferlement par dépassement du seuil critique de la cambrure. Le déclenchement du déferlement au niveau du premier ventre d'oscillation de la surface libre est un facteur limitant les amplitudes des houles testées. Les conditions les plus défavorables du point de vue de la stabilité des blocs sont ainsi atteintes lorsque le déferlement se produit sur l'ouvrage. Un déferlement en avant de la digue provoque une dissipation intense d'énergie et des efforts moindres sur les blocs. Ce phénomène associé à la réflexion de l'ouvrage sera présent de la même façon sur site.

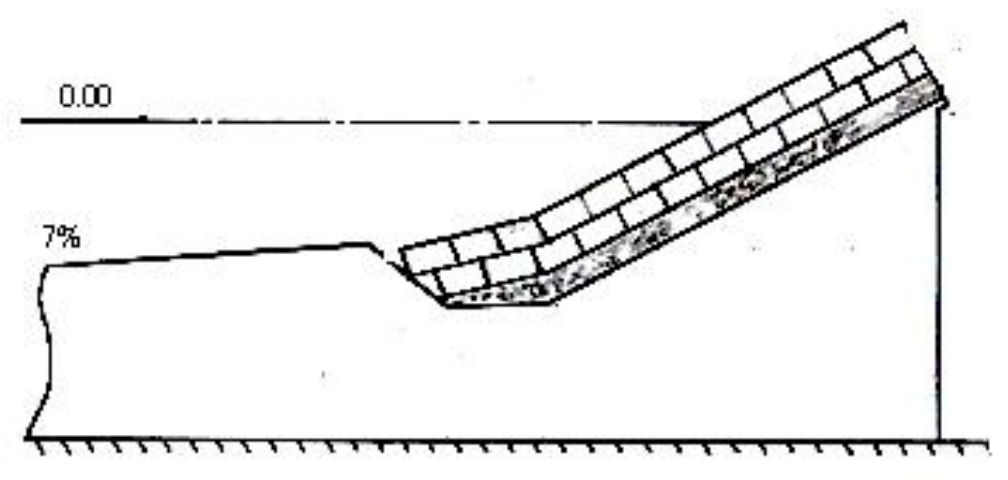

Figure 2 -Disposition du talus

Différentes configurations de talus (cf. figure $\mathrm{N}^{\circ} 3$ ) avec différents types de butée ont faits l'objet de la présente étude. Trois pentes du talus : 2/1, 3/2, 4/3, (dimension horizontale/verticale) ont été testées.

La carapace est formée de blocs de forme parallélépipédique (de tailles 85grammes en modèle pour les 1ères séries d'essais et de 210 grammes ensuite), disposés en deux couches sur une sous couche de gravier de tailles fonction du poids des blocs de carapace, respectivement 10-20 $\mathrm{mm}$ (en modèle) pour les premiers blocs et 20-30 $\mathrm{mm}$ pour les autres

Le pied du talus est fixé successivement aux côtes -3.75 , -5.00 et -6.60 (nature).

La pente du fond devant l'ouvrage est simulée sur une longueur d'environ 170m (au 1/53) avec une pente de $7 \%$. La profondeur d'eau au large est de $0.35 \mathrm{~m}$ en modèle, soit $18,50 \mathrm{~m}$ en nature (échelle1/53). 


\section{Analyse et synthèse des résultats}

L’objectif de la présente étude est d'analyser l'effet de la butée sur la stabilité du talus dans le cas des houles critiques au déferlement. A cet effet et comme indiqué dans le tableau de résultats, 20 séries d'essais avec trois profondeurs d'eau en pied de talus ( $3,75 \mathrm{~m}, 5,00 \mathrm{~m}$, 6,60m), 3 tailles de blocs de carapace et 12 configurations de butées de pied ont été testées.

Pour chaque série d'essais, les houles sont générées par paliers croissants en périodes (10s, 13 s et $16 \mathrm{~s}$ en nature) et en hauteurs jusqu'à l'atteinte du seuil d'instabilité de l'ouvrage (cf. tableau $\mathrm{N}^{\circ} 1$ ).

A noter que le seuil d'instabilité du talus est apprécié par l'observation visuelle des mouvements des blocs et l'analyse des enregistrements vidéo. Le seuil est considéré comme atteint lorsqu'au moins 1 bloc faisant partie de l'élément étudié est soulevé avec un mouvement supérieur à la moitié de sa dimension.

L'analyse du tableau $\mathrm{N}^{\circ} 1$ fait apparaître les points suivants :

(a) Lorsque la butée est encastrée dans une souille rigide, la stabilité du talus n’est pas fragilisée. Le talus tient avec les valeurs critiques au déferlement (essai $\mathrm{N}^{\circ} 4$ )

(b) L'effet de la pente sur le talus est confirmé. On note que pour des conditions d'essais hydraulique et de configuration de talus similaires, le seuil d'instabilité est atteint dès $\mathrm{H}=3,60 \mathrm{~m}$ pour le cas d'une pente de $4 / 3$ (essai $\mathrm{N}^{\circ} 13$ ) alors qu’il n’apparaît qu’à partir de $4,40 \mathrm{~m}$ avec une pente de talus de $2 / 1$ (essai $\mathrm{N}^{\circ} 18$ )

(c) L'érosion du pied de talus joue un rôle important sur la stabilité intrinsèque du talus. Ni l'augmentation relative de la taille des blocs de carapace (essais $\mathrm{N}^{\mathrm{os}} 18$ et 19), ni le "'gabionnage"' des blocs de butée (essais $\mathrm{N}^{\mathrm{os}} 14$ et 15 ) ne donnent lieu à une amélioration sensible du seuil d'instabilité.

(d) Les seuils d'instabilité entraînant la ruine de l'ouvrage décroissent avec le niveau d'eau en pied de talus. Ceci est dû au fait que plus le niveau d'eau devant l'ouvrage est faible plus les vitesses des particules fluides dans la butée sont importantes. Les dépressions conséquentes provoquent le soulèvement du soubassement de la butée, déchaussant ainsi les blocs de la butée qui finissent par se mettre en mouvement. 
Tableau 1 - Résultats des essais

\begin{tabular}{|c|c|c|c|c|c|c|c|}
\hline \multirow[t]{2}{*}{$N^{\circ}$ d'essai } & \multirow{2}{*}{$\begin{array}{l}\text { Talus de } \\
\text { l'ouvrage } \\
\text { (H/V) }\end{array}$} & \multirow{2}{*}{$\begin{array}{l}\text { Profondeur } \\
\text { d'eau en } \\
\text { pied talus }\end{array}$} & \multirow{2}{*}{$\begin{array}{l}\text { Taille des } \\
\text { blocs de } \\
\text { carapace }\end{array}$} & \multicolumn{2}{|c|}{$\begin{array}{l}\text { Hi critique au } \\
\text { large }\end{array}$} & \multirow[t]{2}{*}{ Description particulière des modèles testés } & \multirow{2}{*}{$\begin{array}{l}\text { Talus réalisé } \\
\text { tel que } \\
\text { montré sur } \\
\text { figure } 3\end{array}$} \\
\hline & & & & $\begin{array}{c}T \\
(s)\end{array}$ & $\begin{array}{c}\mathbf{H} \\
(\mathrm{m})\end{array}$ & & \\
\hline $\begin{array}{l}1 \\
2 \\
3 \\
4 \\
5 \\
6\end{array}$ & $3 / 2$ & $\begin{array}{l}3.75 \mathrm{~m} \\
", \\
، \\
\text { ", } \\
6.60 \mathrm{~m} \\
3.75 \mathrm{~m}\end{array}$ & $\begin{array}{l}5.2 \mathrm{~T} \\
", \\
، \\
\text { " } \\
8.3 \mathrm{~T}\end{array}$ & $\begin{array}{l}10 \\
10 \\
16 \\
16 \\
16 \\
16\end{array}$ & $\begin{array}{l}3.50 \\
3.90 \\
4.70 \\
6.30 \\
5.20 \\
4.50\end{array}$ & $\begin{array}{l}\text { Butée apparente sur une souille en gravier 10-20mm } \\
\text { Butée apparente avec la } 1^{\text {ère }} \text { rangée enlevée } \\
\text { Butée ensouillée prolongée avec du gravier 10-20mm } \\
\text { Butée encastrée dans une souille rigide } \\
\text { Butée posée sur gravier 10-20mm (modèle) } \\
\text { Carapace ensouillée }\end{array}$ & $\begin{array}{l}\text { Fig. d } \\
\text { Fig. e } \\
\text { Fig. b } \\
\text { Fig. a } \\
\text { Fig. c } \\
\text { Fig. c }\end{array}$ \\
\hline $\begin{array}{c}7 \\
8 \\
9 \\
10 \\
11 \\
12 \\
13 \\
14 \\
15 \\
16 \\
17\end{array}$ & $4 / 3$ & $\begin{array}{c}\text { 6.60m } \\
\text { 5.00m } \\
" \\
" \\
" \\
3.75 \mathrm{~m} \\
" \\
" \\
" \\
"\end{array}$ & $\begin{array}{c}5.2 \mathrm{~T} \\
" \\
" \\
" \\
" \\
" \\
" \\
" \\
" \\
12.9 \mathrm{~T}\end{array}$ & $\begin{array}{l}13 \\
13 \\
13 \\
16 \\
16 \\
16 \\
16 \\
16 \\
16 \\
16 \\
16\end{array}$ & $\begin{array}{l}6.25 \\
4.50 \\
5.00 \\
6.00 \\
4.85 \\
6.00 \\
3.60 \\
4.40 \\
4.40 \\
5.90 \\
4.30\end{array}$ & $\begin{array}{l}\text { Butée formée de } 2 \text { blocs } 12.9 T \text { sur gravier 10-20mm } \\
\text { Butée sur gravier 10-20mm (modèle) } \\
\text { " gravier 20-40mm (butée stable) } \\
\text { Butée avec } 2 \text { rangées de blocs } 12.9 T \\
\text { " I rangée mais plus ensouillée } \\
\text { Carapace ensouillée } \\
\text { Butée apparente avec blocs liés sur } 3 \text { rangées } \\
\text { Butée apparente avec blocs liés sur } 5 \text { rangées } \\
\text { Butée enfouie avec blocs liés } \\
\text { Carapace ensouillée }\end{array}$ & $\begin{array}{l}\text { Fig. g } \\
\text { Fig. b } \\
\text { Fig. b } \\
\text { Fig. b } \\
\text { Fig. f } \\
\text { Fig. g } \\
\text { Fig. c } \\
\text { Fig. h } \\
\text { Fig. i } \\
\text { Fig. j } \\
\text { Fig. k }\end{array}$ \\
\hline $\begin{array}{l}18 \\
19 \\
20\end{array}$ & $2 / 1$ & $\begin{array}{c}3.75 \mathrm{~m} \\
،, \\
،,\end{array}$ & $\begin{array}{l}5.2 \mathrm{~T} \\
12.9 \mathrm{~T} \\
.\end{array}$ & $\begin{array}{l}16 \\
16 \\
16\end{array}$ & $\begin{array}{l}4.40 \\
4.30 \\
5.00\end{array}$ & $\begin{array}{c}\text { Carapace ensouillée sur gravier 10-20mm (modèle) } \\
\text { " }\end{array}$ & $\begin{array}{l}\text { Fig. b } \\
\text { Fig. b } \\
\text { Fig. k } \\
\text { Fig. I }\end{array}$ \\
\hline
\end{tabular}



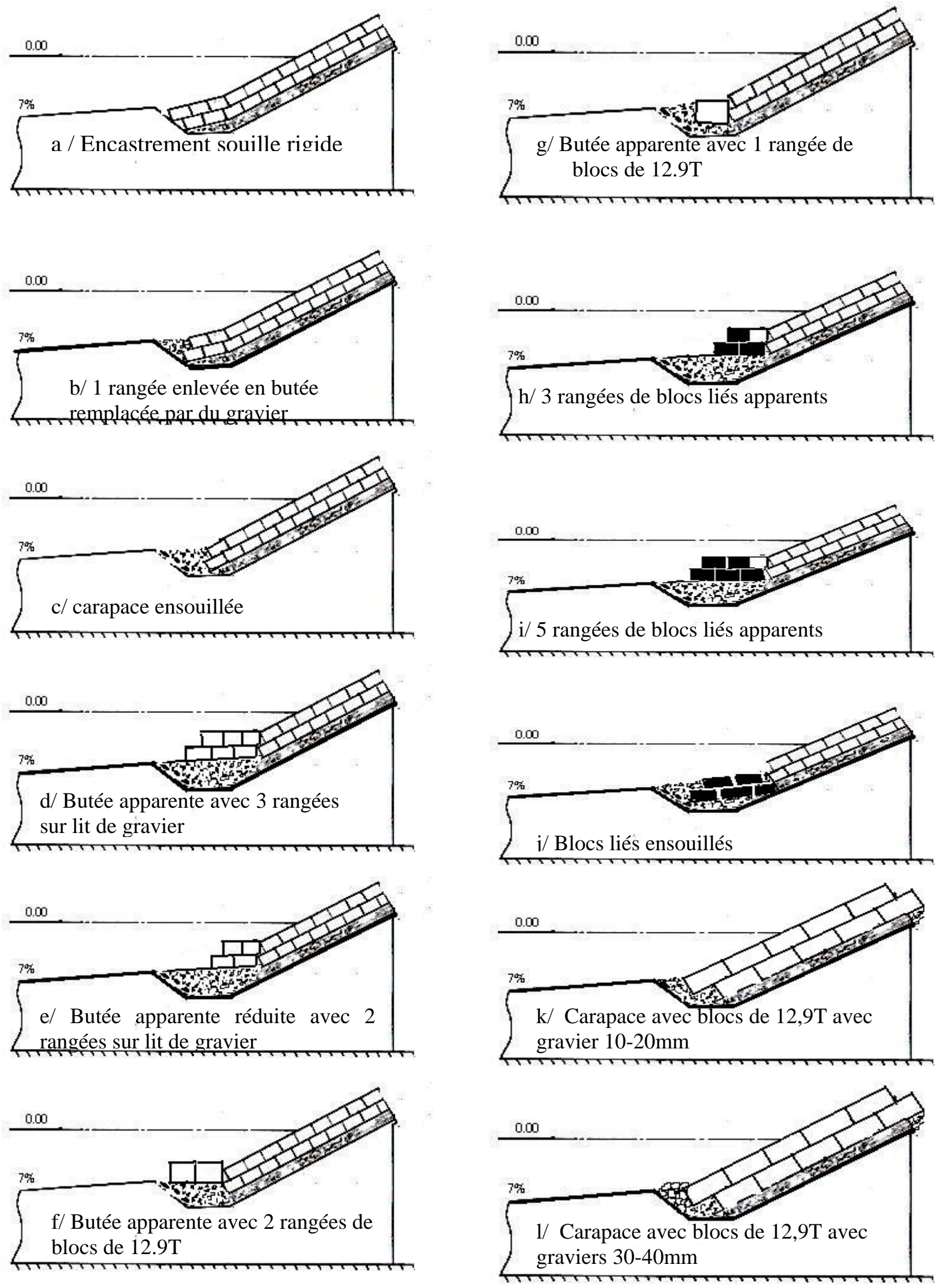

Figure 3 - Schémas des différents types de butées testées en modèle 2D 


\section{Conclusions}

Différentes configurations de butées ont été ainsi testées avec plusieurs catégories de soubassements. La sollicitation de chacune de ces configurations avec des houles croissantes en périodes et en amplitudes a fait apparaître l'effet de la butée de pied sur la stabilité du talus.

Les discontinuités de la pente dues à la présence de la butée ou la '’prise au vent' des blocs apparaissant en saillie en pied de talus apparaissent comme pénalisant pour la stabilité globale. Il a été ainsi noté que dans de tels cas l'augmentation substantielle de la taille des blocs en butée n'est pas toujours la solution pour la stabilité de cette dernière.

Il est recommandé de rechercher à chaque fois le meilleur lissage possible des courbes enveloppe du talus et un soin particulier pour le renforcement du pied de talus dans le cas où le sol de fondation est composé de matériaux meubles.

\section{Références}

1.Arneborg, L., Hansen, E.A., Juhl, J., 1995: Numerical modelling of local scour at partially reflective structures. Final Proceedings of the project rubble-mound breakwater failure modes. MAST 2-CT92-0047, Vol, 2.

2.Fredsoe,J., Sumer, B.M., 1997: Scour at the round head of a rubble-mound breakwater. Coastal Engineering, Vol29 (3), pp.231-262

3.Goda Y., Suzuki Y., 1976: Estimation of incident and reflected waves in random wave experiments. Proc. $15^{\text {th }}$ Coastal Engineering Conference, Hawaii, pp. 828-845

4.Sawaragi, T., 1966: Scouring due to wave action at the toe of permeable coastal structure. Proc. $10^{\text {th }}$ Conference on Coastal Engineering, Tokyo, Japan Vol. 2, pp. 1036-1047.

5.Sumer B.M., Fredsoe J., 2000:- Experimental study of 2D scour and its protection at a rubble-mound breakwater. Coastal Engineering, Vol40(1) pp 59-87

6.Sumer B.M., Whitehouse R.J.S., Torum A., 200: Scour around coastal structures: a summary of recent research. Coastal Engineering 44, pp. 153-190

7.USACE, 2001: Coastal Engineering Manual. US Army Corps of Engineers. Design of coastal projects elements, Part VI. 